\title{
Le rôle du Parlement écossais dans l'examen de la norme communautaire ou la face cachée de la dévolution
}

\section{Elizabeth Gibson-Morgan}

\section{CpenEdition}

\section{Journals}

Édition électronique

URL : https://journals.openedition.org/etudesecossaises/204

DOI : 10.4000/etudesecossaises.204

ISSN : 1969-6337

Éditeur

UGA Éditions/Université Grenoble Alpes

\section{Édition imprimée}

Date de publication : 30 avril 2009

Pagination : 185-200

ISBN : 978-2-84310-138-0

ISSN : 1240-1439

\section{Référence électronique}

Elizabeth Gibson-Morgan, « Le rôle du Parlement écossais dans l'examen de la norme communautaire ou la face cachée de la dévolution », Études écossaises [En ligne], 12 | 2009, mis en ligne le 30 avril 2010, consulté le 11 avril 2023. URL : http://journals.openedition.org/etudesecossaises/204 ; DOI : https://doi.org/10.4000/etudesecossaises.204 


\section{Le rôle du Parlement écossais dans l'examen de la norme communautaire ou la face cachée de la dévolution}

Membre fondateur d'une Union de nations, le Royaume-Uni, proche d'un pacte ou d'un contrat asymétrique fondé sur un processus d'ajustement perpétuel entre le centre et la périphérie, l'Écosse a gagné en autonomie grâce au processus de dévolution mis en place à la fin des années 1990 qui a proposé un nouveau mode de division et de gestion du territoire.

Pendant longtemps, l'Écosse a souffert au sein du Royaume-Uni d'une représentation politique limitée parce que ce dernier s'affirmait, en dépit de sa nature particulière, comme un État unitaire ; aujourd'hui, le Royaume-Uni n'est plus tout à fait unitaire, même si la dévolution n'est ni une régionalisation, à proprement parler, ni un véritable fédéralisme. Le rétablissement du Parlement écossais a permis, dans une certaine mesure, d'y remédier.

La loi sur l'Écosse - Scotland Act 1998 - a, en effet, restauré un Parlement écossais monocaméral, se démarquant par là nettement du Parlement de Westminster à l'origine du bicamérisme ${ }^{1}$. Élu pour quatre ans, fort de 129 membres, le Parlement écossais est doté d'un pouvoir circonscrit au vote de la loi en vertu de l'article 5 du Scotland Act 1998, et d'une autorité limitée en matière d'imposition. Mais si le Parlement de Westminster a délégué ses fonctions, il n'a pas pour autant renoncé à son pouvoir souverain de légiférer pour l'ensemble du pays.

En effet, il a conservé une compétence législative générale sur les sujets relevant des Affaires étrangères, dont l'Union européenne, le Parlement écossais ne pouvant voter sur les matières réservées au Parlement britannique. Peut-on, toutefois, dire que les Affaires européennes relèvent

1. Au sens de l'existence au sein d'un parlement, de deux assemblées distinctes, composées selon des modalités différentes. Sur le plan historique, le bicamérisme est apparu en Angleterre, où le parlement fut composé à partir des XIII ${ }^{\mathrm{e}}$ et XIV ${ }^{\mathrm{e}}$ siècles des barons - Magnum Concilium - et des représentants des communautés - Commune Concilium. 
uniquement de la politique étrangère et par conséquent ne concernent guère les affaires intérieures écossaises?

Paradoxalement, le Royaume-Uni n'a pas jugé nécessaire d'élaborer une politique d'ensemble pour déterminer et encadrer sa politique européenne une fois la dévolution en marche. Seuls deux grands principes ont guidé le pays jusqu'ici, à commencer par le maintien de la position prééminente du gouvernement britannique dans l'élaboration de la politique européenne du pays - l'exécutif britannique détenant une influence bien supérieure à l'assemblée parlementaire du fait de l'intervention des ministres au stade de la négociation des projets de directives européennes. Le second principe, pour sa part, se définit plutôt par la négative, à savoir ne rien faire qui puisse compromettre le fonctionnement du dispositif européen en place, en partie confié à des organes spécialisés dans les Affaires européennes au sein du Parlement qui collaborent étroitement avec le gouvernement britannique. Autrement dit, tout changement doit se faire dans la continuité en respectant le statu quo antérieur à la dévolution, d'où l'idée de faire des Affaires européennes un domaine réservé à l'échelon central.

En définitive, confronté à la diffusion de l'autorité politique du niveau national vers les niveaux infra et supra nationaux, le Parlement britannique a dû se repositionner. Quant à l'Écosse, elle s'efforce de tirer profit de ce paysage national et européen en pleine mutation pour y trouver sa place et y promouvoir au mieux ses intérêts, et cela d'autant plus que les institutions européennes se préoccupent toujours davantage de l'avenir des régions en s'engageant plus fermement en leur faveur, au point d'imaginer le développement de relations directes avec les instances subnationales.

Face à ces évolutions, le Parlement britannique a tenté de s'adapter en organisant très tôt un contrôle de l'activité communautaire du gouvernement par la mise en place des organes spécialisés dans les Affaires européennes. Les commissions pour les Affaires européennes ont donc été imposées par la pratique interne et communautaire en tant que rouage essentiel dans la procédure de participation du parlement national au processus normatif de l'Union européenne, gage d'un meilleur suivi des questions européennes. Dans cette perspective, le rapporteur pour la Commission institutionnelle sur les relations entre les parlements nationaux et le Parlement européen, Monsieur Seeler, insistait en janvier 1989 sur la nécessité de la mise en place de tels organes : 
Il est souhaitable que les parlements nationaux se dotent de commissions européennes de même rang que les commissions parlementaires ${ }^{2}$.

La proposition de Monsieur Seeler a non seulement le mérite de rappeler que le travail en commission est véritablement devenu l'un des moments-clés de la vie parlementaire - y compris pour le Parlement de Westminster où traditionnellement les débats au sein des chambres en séance plénière étaient privilégiés par rapport au travail en commission, au nom du principe de l'unité du Parlement - mais aussi celui de proposer un moyen de renforcer la participation des parlements nationaux au processus communautaire à travers la création de pôles de spécialisation. Faut-il pour autant réserver les Affaires européennes à quelques spécialistes, au risque de relancer la polémique sur une Union européenne limitée à une communauté d'experts ? À travers la création d'instances spécialisées dans les affaires communautaires au sein des parlements nationaux, qui ont toutes un statut de commission de même rang que les autres commissions parlementaires, la construction européenne a contribué à privilégier la mise en place de pôles d'expertise au sein des assemblées.

Ces instances spécialisées sont chargées d'assurer un contrôle de l'action des gouvernements agissant en tant que législateurs au sein du Conseil de l'Union européenne. Parallèlement une autre question fondamentale se pose à ce stade de la réflexion : comment est-il possible d'optimiser le travail des commissions spécialisées dans les Affaires européennes tout en respectant les prérogatives des exécutifs nationaux?

En effet, pour que le contrôle exercé par le biais des commissions spécialisées dans les Affaires européennes puisse être efficace, il doit se faire le plus en amont possible, c'est-à-dire, dès que la Commission européenne fait des propositions au Conseil - et il dépend de l'information gouvernementale. Or, en l'état actuel des choses, les propositions normatives de la Commission européenne sont d'abord transmises à la présidence du Conseil de l'Union européenne qui les envoie aux représentants permanents des États membres à Bruxelles, ces derniers les transmettant à leur tour à une institution réceptrice au sein de l'État membre.

Les commissions spécialisées des parlements nationaux sont de plus en plus amenées à jouer un rôle clé dans ce processus. Ainsi, selon les pays, elles sont chargées de la sélection ou de l'examen des documents produits à l'échelon européen. Elles permettent aux parlements nationaux qui ont perdu la maîtrise du pouvoir normatif, de se réapproprier des prérogatives à travers le droit d'être informés par leurs exécutifs nationaux des projets

2. Parlement européen, doc. A2, 348-88 B. 
de législation communautaire, et celui de donner un avis plus ou moins contraignant sur le sujet. Ainsi, les mécanismes institutionnels au sein de la commission des Affaires européennes du Parlement écossais ${ }^{3}$, créée en 1999, révèlent, à travers les prérogatives significatives dont elle dispose, l'influence de son contrôle parlementaire strict sur la norme communautaire et l'ampleur de l'européanisation du Parlement écossais.

\section{Le cadre institutionnel de l'examen parlementaire de la norme communautaire}

Ce qui différencie avant tout le Parlement de Westminster du Parlement écossais tient à deux caractéristiques étroitement liées, la structure monocamérale du second et le pouvoir de ses commissions. Le premier élément explicatif est d'ordre historique, puisque le premier Parlement écossais de 1707 était monocaméral. Cependant, l'histoire à elle seule ne saurait suffire pour comprendre l'adoption du monocamérisme. Ce dernier résulte d'un choix politique de fond, destiné à mettre en évidence un type de légitimité et l'unité du pouvoir politique qui en découle. Étant donné l'absence de seconde chambre pouvant assurer un deuxième examen des textes et jouer le rôle de contre-pouvoir, les commissions vont y remédier en remplissant les fonctions généralement attribuées à une seconde chambre. La complexité et la technicité croissante des problèmes, l'élargissement rapide du champ du droit et de la loi justifient l'intervention de commissions chargées à la fois d'envisager les projets de loi sous un angle nouveau et de procéder à une relecture des textes.

Ainsi, si comme dans tout parlement, le travail parlementaire au sein du Parlement écossais est divisé en séances plénières et en commissions, ces dernières présentent un caractère inédit. En effet, les commissions du Parlement écossais associent des fonctions qui normalement relèvent de commissions différentes - du moins c'est le cas au sein du Parlement de Westminster - dans la mesure où elles sont à la fois des commissions de législation - quelle que soit l'origine du projet - et des commissions d'enquête et de contrôle. Dans ce but, elles peuvent procéder à l'audition d'experts y compris les ministres écossais. L'article 123 du Scotland Act 1998 stipule que le Parlement ou une commission peut convoquer toute per-

3. La commission des Affaires européennes du Parlement écossais - European Committee - a significativement été rebaptisée commission des Affaires européennes et des relations extérieures - European and External Relations Committee - le 5 mars 2003, afin de consacrer l'extension de ses attributions, notamment son pouvoir de signer des accords bilatéraux avec les autres régions européennes. Le volet européen de cette commission faisant l'objet de la présente étude, l'appellation d'origine a été ici délibérément conservée. 
sonne afin d'apporter un témoignage sur n'importe quel sujet pour lequel un membre de l'exécutif dispose d'une responsabilité générale. En outre, les commissions peuvent inviter des experts à venir apporter un éclairage technique sur les politiques de l'exécutif.

L'ensemble des règles et des procédures qui forment le règlement intérieur prévoit - règle 6 - la mise en place de huit commissions permanentes, dont les domaines d'expertise sont l'audit, l'égalité des chances, les relations européennes et extérieures, les finances, les pétitions publiques, les procédures, la déontologie et les mesures réglementaires visant l'application des lois. En dehors de ces huit commissions permanentes, le Parlement écossais peut décider de constituer toute autre commission jugée nécessaire à l'examen d'un sujet ou d'un domaine particulier - les subject committees ${ }^{4}$. Une commission peut former des sous-commissions et peut se réunir conjointement avec d'autres commissions; en général, les députés écossais qui ne sont pas membres d'une commission peuvent participer aux débats mais ne peuvent pas voter. Une commission est composée généralement de cinq à quinze députés choisis dans le respect de l'équilibre des divers partis et groupes politiques au sein du Parlement.

Officiellement créée le 23 mai 1999, même si elle ne tint sa première séance qu'un mois plus tard, le 23 juin 1999, la commission des Affaires européennes du Parlement écossais était composée initialement de treize membres - réduits depuis à dix ${ }^{5}$ si l'on compte le secrétaire - Stephen Imrie - et son assistant - David Simpson. La commission des Affaires européennes, nommée pour la durée de la session parlementaire, comprend, en effet, deux membres à plein temps : le secrétaire et son assistant. Une fois la dissolution du Parlement et des commissions effective, l'élément de liaison entre l'exécutif écossais et la commission est son secrétaire qui conserve son poste et assure ainsi la continuité du travail de la commission - y compris par la publication des rapports. De plus, la commission des Affaires européennes est dirigée depuis septembre 2005 par un président, ou plutôt une présidente, Linda Fabiani, - poste d'autant plus important que le président possède un rôle d'impulsion. L’idée de départ était que la commission, dont les réunions sont généralement publiques, soit ouverte à tous - même s'ils ne participent pas au vote - afin de constituer un vaste forum où devaient être conviés entre

4. Les subject committees sont des commissions de réflexion qui permettent aux membres qui partagent une préoccupation commune relative à une politique ou à un dossier d'en débattre.

5. Composition de la commission des Affaires européennes (septembre 2005) : Linda Fabiani (présidente), Irène Oldfather (vice-présidente), Dennis Canavan, John Home Robertson, Gordon Jackson, Margaret Ewing, Phil Gallie, Jim Wallace. 
autres les députés écossais, les membres du Comité des régions, les membres du Comité économique et social. Initialement, elle devait se réunir deux fois par semaine - rencontre limitée depuis à une séance hebdomadaire, le mardi.

La commission des Affaires européennes du Parlement écossais est dotée de prérogatives qui, dans le cadre d'un parlement bicamériste, appartiennent normalement à la seconde chambre. À l'instar d'une seconde chambre, elle dispose d'un pouvoir d'initiative en matière législative et peut donner des avis sur le contenu des projets de loi. Sa compétence de contrôle s'exerce à l'égard de l'exécutif écossais ; dans ce but, elle procède à des enquêtes, elle établit des rapports qui contiennent un certain nombre de recommandations et elle les soumet au Parlement écossais. Son mode de fonctionnement dans le traitement des Affaires européennes est, en réalité, très proche de la commission spécialisée dans les Affaires européennes de la Chambre des Lords. Comme cette dernière, elle possède plus qu'un rôle d'instruction puisqu'elle peut délibérer sur toutes les propositions communautaires et faire des rapports sur celles qui soulèvent le plus de questions importantes. Toutefois, le cadre institutionnel de l'examen parlementaire qu'elle exerce demeure assez flou, ce qui d'un coté laisse beaucoup d'ambiguïtés à clarifier ultérieurement, mais ce qui, de l'autre contribue à renforcer l'autonomie du Parlement écossais.

\section{A. Division des compétences entre Westminster et Holyrood : les Affaires européennes, un domaine réservé}

Dans les textes introductifs à la dévolution, les références à l'Europe sont rares et, lorsqu'elles existent, sont définies de manière négative. En effet les textes fondateurs, c'est-à-dire le Livre blanc sur le Parlement écossais de $1997^{6}$ et le Scotland Act 1998, de même que les accords informels et les mémorandums qui les ont suivis, contiennent des dispositions ambiguës en ce qui concerne les relations entre le Parlement écossais et l'Union européenne. L'annexe 5 au Scotland Act 1998, dans sa première partie, article 7, alinéa 1 consacrée aux Affaires étrangères précise :

Les relations internationales, y compris les relations avec les territoires extérieurs au Royaume-Uni, les Communautés européennes (et leurs institutions) ainsi que les autres organisations internationales, la réglementation du commerce international, l'aide au développement et la coopération internationale sont des domaines réservés.

6. Scotland's Parliament, Cm 3658, HMSO, 1997. 
Plus précisément, si la formulation de la position officielle du Royaume-Uni et la négociation avec les partenaires membres de l'Union européenne sont de la responsabilité de l'autorité centrale, l'examen des propositions de l'Union européenne en matière législative et l'application de la politique de l'Union européenne relèvent de la compétence du Parlement écossais. Si toute action de politique étrangère se rattache effectivement à l'activité diplomatique, il semble difficile de considérer la négociation communautaire comme appartenant à la politique étrangère étant donné que les principes de primauté, d'effet direct, et d'invocabilité reviennent à assimiler les normes communautaires au droit interne. En revanche, il ne fait aucun doute que le Parlement de Westminster peut continuer à légiférer dans les domaines dévolus au Parlement écossais, en vertu de l'article 27 alinéa 7 du Scotland Act 1998. En outre, ce dernier, dans son article 28, dresse une énumération négative des matières dévolues qui sont ainsi celles qui ne sont pas réservées au Parlement de Westminster. Le Parlement écossais se voit ainsi opposer un certain nombre de restrictions dont, tout d'abord, le fait que la loi écossaise doit se cantonner aux fonctions et aux lois ayant un rapport avec l'Écosse. Mais surtout, il ne peut intervenir dans les matières explicitement réservées au Parlement de Westminster comme la monarchie, la création et le financement des partis politiques, les affaires étrangères, le service public, la défense nationale, autant de symboles de souveraineté et de pouvoirs régaliens.

En définitive, les nouvelles dispositions institutionnelles qui encadrent désormais les relations européennes de l'Écosse au sein du RoyaumeUni inscrites dans le Livre blanc sur le Parlement écossais de 1997 et le Scotland Act 1998, n'introduisent que peu de changements substantiels en ce qui concerne les Affaires européennes au nom de la volonté de maintenir le statu quo ante. Cependant, le Parlement écossais est tenu, pour sa part, de respecter le droit communautaire et d'appliquer la législation communautaire dans les domaines qui lui sont dévolus. À cette fin un dispositif assez souple a été mis en place.

\section{B. Réglementation des procédures entre exécutif britannique et exécutif écossais : les mécanismes de prévention des conflits}

Le Livre blanc de 1997 sur le Parlement écossais avait envisagé l'élaboration de concordats - ou accords informels - contenant des dispositions visant à établir entre le gouvernement issu de la dévolution et le gouvernement central un modus vivendi portant sur l'échange d'informations ainsi que la notification anticipée des documents communautaires. Ces accords doivent réglementer les domaines réservés lorsque les administrations territoriales y possèdent un intérêt légitime, et réciproquement lorsque le 
gouvernement britannique possède un intérêt dans les domaines décentralisés. Le 6 octobre 1999 fut publiée une déclaration d'intention formalisant le principe d'accords, signée par le gouvernement britannique et chacun des organes décentralisés.

Ces accords informels sont essentiellement de deux types. Les premiers ont pris la forme d'accords multilatéraux, signés par le gouvernent britannique et l'ensemble des organes décentralisés. Ils portent sur des dispositions visant à résoudre les différends entre les administrations, l'implication des administrations décentralisées dans la politique européenne du Royaume-Uni, l'aide financière à l'industrie, la coordination de la politique européenne et des relations internationales, l'échange de statistiques. Les seconds sont bilatéraux, c'est-à-dire qu'ils lient un organe décentralisé et le gouvernement britannique. Qu'ils soient multilatéraux ou bilatéraux, ces accords n'ont aucun caractère contraignant pour leurs signataires : ils constituent plutôt une sorte de code de conduite entre le gouvernement central et les organes décentralisés, ils encadrent l'élaboration de politiques communes. Ils interviennent chaque fois qu'il existe un risque de chevauchement des compétences pour éviter la paralysie des institutions. Donald Dewar, dans une note adressée au Scottish Office en 1998, proposait de les définir de la manière suivante :

Accords passés entre l'exécutif écossais et les départements ministériels du gouvernement du Royaume-Uni, ces accords, non statutaires, ou dispositions purement administratives, sont désignés sous le terme de concordats.

Ces accords doivent garantir en particulier à l'exécutif écossais et au gouvernement central, un déroulement harmonieux des relations de travail en procédant aux consultations nécessaires. Ils doivent, en outre, permettre à l'exécutif écossais d'émettre des propositions dans les sujets non réservés et réciproquement pour le gouvernement central dans les domaines réservés.

Au total, ce cadre général assez souple destiné à favoriser les échanges est fondé sur le bon vouloir réciproque des différentes instances - John Loughlin n'hésite pas à employer pour les décrire l'expression de gentleman's agreement $^{7}$. Ces accords prévoyaient, dans le même esprit de prévention des conflits, la mise en place d'un Comité interministériel ${ }^{8}$ où devaient se dérouler des discussions présidées par le ministre des Affaires étrangères du gouvernement britannique, avec la participation des ministres

7. John Loughlin, «La dimension européenne de la dévolution », Pouvoirs Locaux, n 49 II, 2001, p. 117.

8. Un comité interministériel est généralement une réunion, sous l'autorité du Premier ministre, d'un certain nombre de ministres appelés à débattre d'un sujet déterminé. 
ou des hauts fonctionnaires des assemblées territoriales. Mais le manque de transparence dans son fonctionnement et l'absence de représentants du Parlement de Westminster ont entravé son fonctionnement et entamé la confiance des organes décentralisés à son égard.

Néanmoins, l'idée majeure qui sous-tend l'ensemble de ce dispositif est d'encourager des relations d'exécutif à exécutif et de parlement à parlement.

\section{Le déroulement de l'examen parlementaire : un contrôle parlementaire strict mais non contraignant de la norme communautaire}

Les parlements - dont le Parlement écossais - se sont efforcés de s'adapter aux nouveaux modes de gouvernance européenne par un regain de leur activité de contrôle, notamment à travers la spécialisation fonctionnelle de leurs élus dans le cadre des commissions permanentes des Affaires européennes. Plus importante encore que cette spécialisation est la portée juridique du contrôle que la commission des Affaires européennes du Parlement écossais exerce vis-à-vis de l'exécutif écossais en matière européenne, car s'il n'est pas contraignant, ce contrôle en revanche s'avère strict.

\section{A. Un contrôle parlementaire strict}

La commission des Affaires européennes du Parlement écossais est soumise à des règles de fonctionnement fixées par la règle 6 alinéa 8 du règlement intérieur du Parlement. Cet organe spécialisé joue d'abord un rôle important dans la phase de réception et d'instruction des textes communautaires.

1. Réception des propositions communautaires par la commission des Affaires européennes du Parlement écossais : examen ex ante.

Il est, à ce stade, essentiel que la commission soit informée dans de bonnes conditions en termes de délais comme de contenu par le gouvernement britannique, sur lequel repose une obligation préalable de transmission des projets de directives et de règlements de l'Union européenne, ce qui signifie une obligation d'information et de consultation du Parlement écossais sur les enjeux européens. Le gouvernement britannique est, en effet, tenu de fournir à la commission des Affaires européennes le plus tôt possible une information complète sur tout sujet susceptible d'intéresser l'Écosse. Les documents européens ainsi transmis sont, de plus, accompagnés d'un mémorandum comprenant un résumé de la proposition de 
la Commission européenne, ses implications juridiques et politiques, le calendrier de son examen et la position gouvernementale à leur sujet. Le gouvernement britannique doit transmettre ces documents au même moment qu'il les transmet au Parlement de Westminster. Les mémorandums sont d'une grande utilité pour les membres de la commission des Affaires européennes étant donné qu'ils exposent officiellement le point de vue du gouvernement d'une part, et qu'ils aident à la sélection des projets importants d'autre part.

La commission des Affaires européennes, contrainte par des délais très courts, n'a d'autre choix que de s'en remettre à l'analyse du gouvernement britannique. Ce dernier reçoit quelque 8000 documents par an des institutions européennes sur une grande variété de sujets dont 1200 sont transmis au Parlement de Westminster - 800 d'entre eux feront l'objet d'un examen détaillé. En conséquence, la commission des Affaires européennes devra procéder pour sa part à un tri très sélectif - elle examine quelque 200 documents par an - et jouera un rôle central dans le tri des projets jugés les plus importants. Une des principales difficultés rencontrées par la commission des Affaires européennes dans l'exercice de ses fonctions est la brièveté des délais qui lui sont impartis pour procéder à l'examen au fond des textes communautaires. Contrairement à la France où, à l'origine, seules les propositions d'actes communautaires comportant des dispositions de nature législative étaient transmises par le gouvernement à l'organe spécialisé, la commission des Affaires européennes du Parlement écossais est saisie de l'ensemble des projets d'actes européens ainsi que d'une grande partie des communications et des rapports de la Commission européenne tels que Livres verts et Livres blancs. Il est intéressant de noter, à ce sujet, que la commission des Affaires européennes intervient de plus en plus au stade pré-législatif, très en amont, ce qui lui permet d'intervenir ex ante, avant la prise de décision communautaire - afin de tenter d'influencer la position et les décisions du gouvernement britannique. En outre, son examen élargi des propositions communautaires lui permet d'avoir une vision d'ensemble de la future législation communautaire et européenne.

Le champ d'action de la commission est très large, dans la mesure où elle est chargée d'examiner la législation européenne qui doit être incorporée à la législation écossaise ainsi que de débattre des affaires européennes pouvant avoir un impact sur les autres commissions ou sur l'Écosse en général. La règle 6 du règlement du Parlement écossais définit sa mission en ces termes :

La commission est chargée d'examiner et de rendre compte des propositions en matière de législation des Communautés européennes, de l'application 
de la législation européenne, et de tout sujet relatif aux Communautés européennes ou à l'Union européenne.

C'est à la commission des Affaires européennes qu'il revient de déterminer la suite à donner à l'examen des textes communautaires. Son contrôle ne pourra être réellement efficace que si, à son examen ex ante, s'ajoute une prise de position juridiquement influente ex post.

\section{2. Élaboration d'une position ex post sous forme de rapport.}

À ce stade, la commission des Affaires européennes est plus particulièrement chargée de déterminer si les textes qui lui ont été transmis présentent un intérêt spécifique pour l'Écosse. Elle dispose pour cela de trois grandes options parmi lesquelles elle devra choisir la plus appropriée. Elle peut décider de transmettre le document européen au Parlement écossais pour un débat en séance plénière sans procéder à son examen en son sein. Une autre possibilité consiste à transmettre le document en question non pas au Parlement écossais dans son ensemble, mais à celle des commissions permanentes jugée la plus compétente en ce domaine. Enfin, elle pourra décider de procéder elle-même à l'examen du document en raison de son caractère urgent ou parce qu'il est de portée générale. Quelle que soit l'option retenue, la commission est soumise à un double impératif : celui de la gestion du temps et celui de la nature de l'examen, rapide ou approfondi en fonction de l'urgence.

Lorsque la commission des Affaires européennes a examiné les projets de directives ou de règlements européens puis a pris position à leur égard, son secrétaire va transmettre au secrétariat de l'exécutif écossais les conclusions de la commission afin qu'il les transmette à son tour à la branche de l'exécutif écossais compétente. Ces conclusions - reflétant la position écossaise en matière européenne - peuvent être alors incorporées par le secrétariat de l'exécutif écossais à l'exposé écossais sur l'Europe - Scottish European Brief - et à la note de couverture écossaise - Scottish cover note - transmise par le secrétaire d'État pour l'Écosse au gouvernement britannique. Le rapport de la commission des Affaires européennes est transmis aux ministres écossais dans l'espoir qu'ils entretiennent un contact étroit avec leurs homologues du Royaume-Uni et se chargent de promouvoir la position de l'Écosse à Londres. C'est le secrétaire d'État - Secretary of State - pour l'Écosse qui représente les intérêts écossais dans les domaines réservés - règle 14, 4.12 - et qui, par conséquent, va jouer ici un rôle déterminant. Les ministres de l'exécutif écossais pouvant être invités à participer aux réunions du Conseil des ministres de l'Union européenne, cela leur permettra de répercuter directement ces conclusions au niveau européen. Ainsi, l'efficacité de cette procédure repose sur une collaboration étroite 
entre la commission des Affaires européennes et l'exécutif écossais dans l'examen des propositions de l'Union européenne afin d'infléchir, dans la mesure du possible, la position du gouvernement britannique.

En outre, la commission des Affaires européennes peut attirer l'attention du Parlement écossais sur toute question européenne, même en l'absence de proposition formelle. Elle constitue, de la sorte, un véritable forum puisqu'elle a la possibilité d'ouvrir un débat, non seulement au sein du Parlement écossais, mais aussi à l'échelle de l'Écosse par le biais d'un vaste processus consultatif.

L'efficacité indéniable du travail de la commission ne saurait en masquer quelques faiblesses non négligeables, notamment le fait que le contrôle exercé par la commission des Affaires européennes sur les textes européens ne lie le gouvernement britannique par aucun mandat juridique.

Aussi, malgré le renforcement de la commission des Affaires européennes du Parlement écossais, tous les moyens ne sont pas encore mis à sa disposition pour lui permettre de réaliser un travail pleinement efficace.

\section{B. Un contrôle non contraignant ou les limites du contrôle parlementaire}

La commission des Affaires européennes du Parlement écossais, en réponse à la publication par la Commission européenne d'un Livre blanc sur la gouvernance européenne ${ }^{9}$ - entendue au sens de mode complexe de prise de décision et de mise en œuvre de celle-ci - a consacré le neuvième rapport de la session 2001 à « La gouvernance de l’Union européenne et à l'avenir de l'Europe : quel rôle pour l'Écosse ? », son objectif consistant à remédier à l'insuffisance de pratiques démocratiques dans le traitement des affaires européennes, et au-delà dans celle du fonctionnement des institutions européennes elles-mêmes. La commission des Affaires européennes, soucieuse de favoriser la transparence des procédures afin de faciliter non seulement l'information mais aussi la participation effective des citoyens, à commencer par la population écossaise, n’a pas manqué de mettre en exergue ce qu'elle juge être deux entraves importantes à l'exercice de son contrôle parlementaire des propositions européennes.

1. L'absence de maîtrise des sources d'information.

S'il existe de nombreuses références dans le droit anglais à l'obligation pour le gouvernement central de procéder, par l'intermédiaire de ses ministres, à des consultations des organes qu'il estime représentatifs des

9. Commission européenne, Livre blanc sur la gouvernance européenne, 2001. 
pouvoirs locaux, celles-ci se limitent souvent à un pur exercice formel. Le gouvernement britannique, bien que cela ne soit pas nécessairement dans son intérêt, peut être tenté de se soustraire à son obligation d'informer la commission des Affaires européennes, d'autant plus que celleci ne possède aucun pouvoir de contrainte ou de sanction. En effet, elle ne peut en aucun cas dicter sa conduite au gouvernement britannique. Plus préoccupant, le mécanisme de réserve parlementaire - dont bénéficient les organes spécialisés dans les Affaires européennes du Parlement de Westminster - garantit que dans la plupart des cas, la commission spécialisée disposera d'un délai utile pour examiner les propositions d'actes communautaires, or ce mécanisme fait cruellement défaut à l'Écosse qui n’a pas manqué de le dénoncer dans son neuvième rapport.

\section{L'absence d'une « réserve d'examen parlementaire » pour l'Écosse.}

Ce mécanisme a été décrit de la manière suivante dans le Livre blanc portant sur l'examen des affaires européennes - The Scrutiny of European Union Business - publié par le gouvernement britannique en novembre 1993 :

Dès lors qu'une proposition de résolution a été déposée, la représentation permanente nationale auprès de l'Union européenne doit demander le report de l'adoption de l'acte communautaire en question à un ordre du jour ultérieur du Conseil ou subordonner le vote définitif du pays de la proposition d'acte communautaire à une prise de position du parlement national ${ }^{\mathbf{1 0}}$.

Cette pratique fait donc obligation au gouvernement britannique de s'opposer à l'adoption d'un texte par le Conseil des ministres tant que le Parlement n'en a pas terminé l'examen, à l'exception de certains cas ; le gouvernement britannique peut, en effet, invoquer des raisons spéciales, autrement dit, lorsqu'un vide juridique doit impérativement être comblé, ou lorsqu'il est essentiel qu'un texte bénéfique pour le Royaume-Uni aboutisse dans des délais très brefs. La réserve est levée pour les projets d'actes qui ne sont pas jugés politiquement ou légalement importants par les commissions spécialisées, ce qui fait peser une importante responsabilité sur le processus de sélection des propositions communautaires.

À l'échelle du Royaume-Uni, cette réserve d'examen parlementaire est une pratique ancienne, puisque le gouvernement britannique s'était engagé à la faire jouer dès l'adhésion de la Grande-Bretagne aux Communautés européennes. Significativement, cette réserve est décrite comme la pierre angulaire du contrôle parlementaire - the corner stone of the scrutiny process - dans le Livre blanc du gouvernement britannique

10. Cabinet Office, The Scrutiny of European Union Business, Londres, TSO, novembre 1993, p. 2. 
mentionné ci-dessus. Établie par une résolution de la Chambre des communes du 30 octobre 1980, elle a été complétée et précisée pour cette chambre par une résolution du 24 octobre 1990. Quant à la Chambre des Lords, cette procédure y est entrée en vigueur dès 1983, suivie d'une réforme en date du 6 décembre 1999 qui en a régularisé la pratique. Système très élaboré de contrôle parlementaire, il permet au Parlement de Westminster d'éviter l'adoption de textes dont il n'aurait pas eu le temps de prendre connaissance ou sur lesquels il n'aurait pas eu le temps de prendre position. Le problème est que cette réserve d'examen parlementaire ne s'applique que pour le Parlement de Westminster, la commission des Affaires européennes du Parlement écossais n’en bénéficie pas, ce qui limite l'autorité et donc la portée de son contrôle et de ses prises de position en matière européenne.

Pour y remédier, la commission des Affaires européennes insista également, dans son neuvième rapport, sur la nécessité pour elle d'être informée sur le déroulement des séances et surtout sur la prise de décision au sein du Conseil des ministres de l'Union européenne. Pour améliorer la qualité de son suivi des Affaires européennes, elle demande donc d'être informée sur l'état des négociations en cours afin, le cas échéant, d'adapter son rythme de travail aux dossiers étudiés par l'Union européenne et d'avoir une vision d'ensemble de la politique de l'Union européenne.

En outre, la mission de la commission des Affaires européennes du Parlement écossais ne saurait se concevoir et ne peut être pleinement efficace sans une ouverture sur l'extérieur lui permettant de dépasser une approche exclusivement écossaise des questions européennes qu'elle a à traiter. En tant qu'agent de liaison, la commission des Affaires européennes, présente au sein du système politique infranational et représentée à Bruxelles, pourrait contribuer à renforcer le lien entre l'Union et les citoyens européens en développant des stratégies de coopération transnationale, en adoptant des positions susceptibles d'introduire l'enjeu européen en Écosse.

Dans cette perspective, elle a noué des relations horizontales avec les autres organes spécialisés, non seulement au sein du Parlement de Westminster - même si elles restent informelles - mais au-delà, avec ses homologues européens, essentiellement par le biais de la Conférence des organes spécialisés dans les affaires communautaires (COSAG). Dotée de compétences accrues depuis le traité d'Amsterdam, la COSAC - au sein de laquelle la commission des Affaires européennes est représentée - tend à fonctionner de plus en plus comme un véritable organe de contrôle parlementaire, étant donné qu'elle s'efforce de suivre au plus près le contenu des travaux du Conseil européen et de la Commission. Elle peut également présenter toute contribution qu'elle juge appropriée sur les 
activités de l'Union, comme l'application du principe de subsidiarité ${ }^{\mathbf{1 1}}$. Elle permet, en particulier, aux organes spécialisés dans les Affaires européennes de s'associer et de se prononcer à la source sur certains actes de l'exécutif communautaire.

La commission des Affaires européennes est également présente au sein du Comité des régions, crée par le traité sur l'Union européenne - ou traité de Maastricht - qui n'est pas une institution communautaire, mais un organe consultatif composé de représentants des collectivités régionales et locales, désignés par les États. Il est obligatoirement consulté dans les cas prévus au Traité et il peut également émettre des avis. Les régions ne pouvant elles-mêmes accéder au rang de partenaires sans avoir été désignées par l'État-membre, le Comité des régions leur accorde une participation institutionnelle au processus communautaire à titre consultatif.

Mais c'est surtout sa participation à l'antenne du Parlement écossais, Scotland Europa, ouverte à Bruxelles en mai 1992 qui permet à la commission des Affaires européennes du Parlement écossais d'obtenir directement des institutions communautaires des informations avant même la publication des documents officiels.

En définitive, le renforcement du contrôle démocratique auquel la commission des Affaires européennes contribue activement est une condition nécessaire pour que l'Union européenne reçoive, de la part des citoyens, l'adhésion sans laquelle elle ne serait qu'une construction technocratique sans grande perspective d'avenir. L'Europe ne pourra se faire sans les citoyens, ou contre eux, à commencer par les Écossais.

\section{Références bibliographiques}

\section{Ouvrages généraux}

BLANC Didier, Les parlements européen et français face à la fonction législative communautaire : aspects du déficit démocratique, Paris, l'Harmattan, 2004.

Bulmer Simon, Burch Martin, et al., British Devolution and European PolicyMaking, Basingstoke, Palgrave, 2002.

Castria Olivier, Quels partenariats pour les régions de l'Union européenne? Rennes, Apogée, 2002.

Givardi, Christian, L'Écosse contemporaine, Paris, Ellipses, 2002.

11. Le principe de subsidiarité s'applique aux compétences partagées entre Communauté, États et Régions, afin d'encadrer leur exercice. C'est un élément de régulation qui permet d'éviter une dépossession systématique des autorités inférieures. 
Costa, Olivier, Vers un renouveau du parlementarisme en Europe? Bruxelles, Université de Bruxelles, 2004.

HourqueBIE Fabrice, Les organes spécialisés dans les affaires communautaires des parlements nationaux, Paris, l'Harmattan, 1999.

Leruez Jacques, L'Écosse: Vieille Nation, Jeune État, Crozon, Armeline, 2000.

Leydier Gilles, La question écossaise, Rennes, Presses Universitaires de Rennes, 1998.

Nuttens Jean-Dominique \& SiCard François, Assemblées parlementaires et organisations européennes, Paris, La documentation française, 2000.

SAurugger Sabine, Les modes de représentation dans l'Union européenne, Paris, l'Harmattan, 2003.

Schnapper Pauline, La Grande-Bretagne et l'Europe : le grand malentendu, Paris, Presses de Sciences-Po, 2000.

\section{Articles}

Costa Olivier \& Latek M., « Paradoxes et limites de la coopération interparlementaire dans l'Union européenne », Journal of European Integration, 2001, $\mathrm{n}^{\circ}$ 23, p.139-164.

LATEK M., «Le poids des traditions parlementaires nationales dans le développement de la coopération interparlementaire : la participation française et britannique à la COSAC », Politique européenne, 2003, $\mathrm{n}^{\circ} 9$, p.143-163.

Loughtin John, « La dimension européenne de la dévolution », Pouvoirs Locaux, 2001, n 49, p.115-120.

\section{Documents institutionnels}

European Committee, Report on Scrutiny of European Documents, SP Paper 33, $1^{\mathrm{er}}$ rapport, 1999.

European Committee, Report on the Governance of the European Union and the Future of Europe: What role for Scotland?, SP Paper 466, 9 rapport, 2001.

External Liaison Unit, Scotland and Europe: A European Seminar, SP Paper 341, février 2001. 